\title{
BMJ Open Effects of closure of an urban level I trauma centre on adjacent hospitals and local injury mortality: a retrospective, observational study
}

\author{
Marie Crandall, ${ }^{1}$ Douglas Sharp, ${ }^{2}$ Xiong Wei, ${ }^{3}$ Avery Nathens, ${ }^{3}$ Renee $\mathrm{Y} \mathrm{Hsia}{ }^{4}$
}

\begin{abstract}
To cite: Crandall M, Sharp D, Wei $X$, et al. Effects of closure of an urban level I trauma centre on adjacent hospitals and local injury mortality: a retrospective, observational study. BMJ Open 2016;6:e011700. doi:10.1136/bmjopen-2016011700
\end{abstract}

- Prepublication history and additional material is available. To view please visit the journal (http://dx.doi.org/ 10.1136/bmjopen-2016011700).

Received 26 February 2016 Revised 20 April 2016 Accepted 22 April 2016

\footnotetext{
${ }^{1}$ Department of Surgery, University of Florida College of Medicine, Jacksonville, Florida, USA

${ }^{2}$ Mental Health Services and Policy Program, Northwestern University, Chicago, Illinois, USA

${ }^{3}$ Department of Surgery, Sunnybrook Health Sciences Centre, Toronto, Ontario, Canada

${ }^{4}$ Department of Emergency Medicine and Philip R. Lee Institute for Health Policy Studies, University of California, San Francisco, San Francisco, California, USA
}

Correspondence to Dr Marie Crandall; marie.crandall@jax.ufl.edu

\section{ABSTRACT}

Objective: To determine the association of the Martin Luther King Jr Hospital (MLK) closure on the distribution of admissions on adjacent trauma centres, and injury mortality rates in these centres and within the county.

Design: Observational, retrospective study.

Setting: Non-public patient-level data from the state of California were obtained for all trauma patients from 1999 to 2009. Geospatial analysis was used to visualise the redistribution of trauma patients to other hospitals after MLK closed. Variance of observed to expected injury mortality using multivariate logistic regression was estimated for the study period.

Participants: A total of 37131 trauma patients were admitted to the five major south Los Angeles trauma centres from the MLK service area between 1999 and 2009.

Main outcome measures: (1) Number and type of trauma admissions to trauma centres in closest proximity to MLK; (2) inhospital injury mortality of trauma patients after the trauma centre closure.

Results: During and after the MLK closure, trauma admissions increased at three of the four nearby hospitals, particularly admissions for gunshot wounds (GSWs). This redistribution of patient load was accompanied by a dramatic change in the payer mix for surrounding hospitals; one hospital's share of uninsured more than tripled from $12.9 \%$ in 1999 to $44.6 \%$ by 2009 . Overall trauma mortality did not significantly change, but GSW mortality steadily and significantly increased after the closure from $5.0 \%$ in 2007 to $7.5 \%$ in 2009 .

Conclusions: Though local hospitals experienced a dramatic increase in trauma patient volume, overall mortality for trauma patients did not significantly change after MLK closed.

\section{INTRODUCTION}

Trauma is the leading cause of death for all Americans aged 1-44 years, claiming more than 180000 lives annually, and injury is a leading cause of death and disability among

\section{Strengths and limitations of this study}

- The study used geospatial analysis to identify a boundary of 32 contiguous zip codes to define the Martin Luther King Jr Hospital (MLK) service area, which contained over $80 \%$ of the trauma admissions to MLK during the study period.

- Zip codes were used instead of predetermined drawn catchment areas to simplify the quantitative data analysis.

- The analysis captured the years leading up to the MLK closure, the closure transition period including trauma centre de-designation, and 2 years after the hospital closure.

- The study is unique to one particular trauma system in a local context, and thus, the findings cannot be extrapolated to all areas of the country that have experienced closures of trauma centres.

children and adults. ${ }^{1}$ There are currently more than 1000 trauma centres in the USA, which are hospitals that have committed resources to the care of the injured patient. California trauma centres are designated as levels I-IV. All centres must have a multidisciplinary trauma team and an emergency department (ED), and all must have personnel, services and equipment necessary to care for trauma patients. Trauma centres, as part of organised trauma systems, have been shown to improve injury mortality by $10-20 \%$ compared with areas that do not have specialised trauma care. ${ }^{2-4}$

Current literature documenting hospital ${ }^{5} 6$ and ED closures ${ }^{7-10}$ has produced controversial findings; some studies have shown associations with higher mortality due to deteriorating access, ${ }^{7}$ while others have shown no association with poorer outcomes. ${ }^{6}$ Several studies have specifically examined closures of trauma centres, ${ }^{11}{ }^{12}$ but to our knowledge, few have discussed the population effects on outcomes as a result of trauma centre closures. ${ }^{13}$ 
Given the conflicting evidence for hospital and ED closures, and the paucity of evidence of how trauma centre closures specifically affect outcomes, the goal of this study was to focus on one trauma centre closure in a specific context, using it as a case study to show how there may be different effects based on particular contexts. We studied Martin Luther King Jr/Drew Medical Center, currently a multiservice ambulatory care centre in the southwest region of Los Angeles County, serving the communities of Compton, Watts and Willowbrook. ${ }^{14}$ South Los Angeles is a distinct region of Los Angeles County that comprises 25 neighbourhoods and three unincorporated districts. The population is approximately 95\% African American and Latino, and despite being home to the University of Southern California and the Los Angeles Coliseum, the area is generally economically distressed and suffers high rates of trauma, particularly penetrating trauma, such as gunshot wounds (GSWs).

In response to the lack of sufficient access to healthcare and opportunities in this low-income area ${ }^{15}$ the Martin Luther King Jr Hospital (MLK) was opened in 1972, then designated as a level I trauma centre in $1983 .{ }^{16}$ Despite the hospital's assets, a series of highly publicised deaths led to the closure of the cardiac monitoring unit by December 2003, and then MLK's redesignation as a level II trauma centre in February 2004. Trauma centre designation was removed completely from MLK in December 2004, but the inpatient and emergency services remained open in the hopes of improving existing services. By 2006, more patient deaths at MLK were reported as attributable to medical errors, and all inpatient and emergency services were closed in August 2007. Following this closure, a provision plan was created for emergency transport and staffing at nearby public and private hospitals, given that the closure of the second-busiest trauma centre in Los Angeles might adversely affect neighbouring hospitals and local communities. ${ }^{17} 18$ Subsequent impact analyses were also reported, noting challenges and highlighting the need for a comprehensive care plan for the area. ${ }^{18-21}$

This study seeks to fill this gap in literature by evaluating the redistribution of the volume of injured patients in neighbouring hospitals as well as trauma mortality of admitted patients within these hospitals and overall mortality in the county. We hypothesised that the MLK closure in 2007 significantly impacted the volume of trauma patients at other south Los Angeles hospitals, and potentially increased the trauma mortality for south Los Angeles.

\section{METHODS}

\section{Study design and data sources}

We used non-public patient-level data from the California Office of Statewide Health Planning and Development (OSHPD) for all patients admitted to general, acute, non-federal hospitals in the state of California. We limited the data set to all trauma patients in south Los Angeles during the study period from 1999 to 2009 using zip codes for this defined area. We performed an observational, retrospective study of Patient Discharge Data for the years leading up to the MLK closure (1999-2003), the closure transition period including trauma centre de-designation (2004-2007), and 2 years after hospital closure (2008-2009).

\section{Patient population}

We first characterised the patient sample with simple descriptive statistics, including trauma admissions, demographics and injury mortality. We then used geospatial analysis to identify a boundary of 32 contiguous zip codes to define the MLK service area for trauma incidents. This service area contained over $80 \%$ of the trauma admissions to MLK during the study period. We used zip codes instead of predetermined drawn catchment areas to simplify the quantitative data analysis. This boundary included the 31 zip codes that had the largest number of total admissions of any kind to MLK, and the 29 zip codes that had the largest number of trauma admissions to MLK while it was in operation. We did not limit the population by age, though MLK was not a paediatric trauma centre, because patients 15 years and older would have been seen routinely, and younger patients may have been seen due to age uncertainty or self-transport.

Trauma admissions included those defined by the International Classification of Disease, Ninth Revision, Clinical Modification (ICD-9-CM) diagnosis codes 800904.9, 910-929.9 and 950-959.9 in either the principal diagnosis or in any of the 24 secondary diagnoses in our data set $(\mathrm{N}=117161)$, excluding visits with ICD-9-CM codes indicating drowning, burns, bites and stings, overexertion, poisonings, foreign body, suffocation or late effects of injury, as well as those with a sole traumatic ICD-9-CM diagnosis of strains and sprains, or contusions with intact skin surface ${ }^{22}$ (see online supplementary appendix table 1). Burns were excluded because definitive care is provided at LA County-USC, a specialised burn centre. Patients who did not have an injury mechanism as denoted by an E-code (external cause of injury) were excluded $(\mathrm{N}=854)$, leaving a total of 37131 admissions for the analysis.

\section{Main outcome measures}

The primary outcome of interest was the number and type of trauma admissions to trauma centres in closest proximity to MLK. The trauma centres (TCs) within 10 miles of MLK were: TCA (level II, 2.3 miles from MLK), TCB (level I, 7.0 miles from MLK), TCC (level II, 7.9 miles from MLK), and TCD (level I, 9.6 miles from MLK).

The secondary outcome of interest was in-hospital injury mortality of trauma patients after the trauma centre closure. The observed mortality rate was compared with expected mortality rate using a risk-adjusted model incorporating age, injury severity, survival risk, injury mechanism, gender, year, patient insurance status, and race for each hospital, and for the MLK service area. 


\section{Statistical methods}

We used descriptive analyses to evaluate the primary outcome of number and type of trauma admissions to different facilities. For our secondary outcome, predicted mortality for the region was calculated using separate models for each of the most common mechanisms of injury (stab wounds, GSWs, falls and motor vehicle collisions), and overall. Model covariates included age, gender, insurance status, race, ISS $>16$, SRR and year. All these variables are known to have an effect on injury mortality. Insurance status was categorised into self-pay, public and private. Race was categorised into African American, Latino, Asian American and White.

Injury severity was measured by the Injury Severity Score (ISS). We used ICDPIC V.3.0 for Stata V.11 (ICDPIC V.3.0 for Stata. 2008;2011. http://ideas.repec. org/c/boc/bocode/s457028.html). (StataCorp, College Station, Texas, USA) to calculate the ISS from ICD-9-CM codes. We used the accepted standards of categorising injury severity by creating a binary variable denoting severe (ISS >16) and less severe (ISS $\leq 16) .{ }^{23} 24$

Survival risk ratios (SRRs) associated with each of the relevant ICD-9-CM codes were obtained from the American College of Surgeons Trauma Quality Improvement Program (TQIP) for blunt and penetrating trauma. These values were calculated from 2011 nationwide injury survival statistics, then matched to the ICD-9-CM codes recorded for each case. The lowest ratio for each case was then used in the model. Of note, a proportion of diagnostic codes did not match SRRs. We deliberately used nationwide SRRs to have the largest available sample to predict expected outcomes and to decrease any potential impact of regional variability.

We first estimated mortality models for each common injury mechanism and traumas overall in the MLK service area. To do this, we used preclosure mortality data from all hospitals that served the MLK catchment area. We controlled for age, race, gender, insurance status, ISS, SRR and a continuous time variable. ${ }^{25}$ The time variable was included to account for secular trends in trauma incidence, mechanism and mortality rates. We specifically examined mortality for GSWs, as MLK had historically cared for most of the GSW victims in the area, and the effects of MLK closure might be borne disproportionately by this group. We then compared observed mortality rates with rates predicted by the risk-adjusted model for hospitals serving the MLK catchment area. CIs for predicted mortality were calculated based on the SEs associated with the predicted values.

All statistical analyses used Stata V.11 (College Station, Texas, USA). Geospatial analysis was performed using Arc-GIS 10 software (Esri, Redlands, California, USA). This study was approved by the University of California, San Francisco Committee on Human Research, and the California Committee for the Protection of Human Subjects.

\section{RESULTS}

South Los Angeles county trauma volumes across hospitals

Between 1999 and 2009, aA total of 37131 trauma patients were admitted to the five major south Los Angeles trauma centres from the MLK service area. There was no significant change in total annual admissions at the five hospitals over the time period, with 3236 admissions in 1999, to a peak of 3546 in 2003 and down to 3173 by 2009. By contrast, admissions at individual hospitals in the MLK service area experienced marked longitudinal trends. Despite trauma centre catchment area downsizing and eventual de-designation between February and December of 2004, over 1000 trauma patients were seen at MLK in 2004, more than 500 in 2005, and over 450 in 2006. TCD, the busiest trauma centre in the area, had a significant drop in admissions over the study period. However, the three other nearby centres experienced increases in trauma volumes after the MLK closure. TCC, which had not been a trauma centre prior to MLK's closure, but was given a level II designation to increase local capacitance, saw a 10-fold increase in trauma admissions (figure 1).

\section{Demographic and injury severity redistribution across hospitals}

Patient mechanisms of injury at the five area trauma centres were very different from each other during the study period (table 1), with MLK seeing much higher numbers and percentages of penetrating trauma. Several centres saw significant changes after the closure of MLK. TCs A, B and C experienced marked increases in the proportion of GSW admissions after MLK closed; a twofold increase at TCs A and B (approximately 150300 patients annually) and a 10 -fold increase at TCC (from 10 to 300 patients annually), which were all statistically significant ( $p$ values for all $<0.001$ ).

Over the entire time period, injury severity as measured by the proportion of patients with an ISS $>16$ also increased at TCA $(9.1 \%-14.8 \%, \mathrm{p}<0.001)$ and TCC $(3.1 \%-11.1 \%, \mathrm{p}<0.001)$. Gender mix and mean age of trauma patients only changed significantly at TCC, transitioning to an overall younger (mean age dropped from 54.1 to 39.9 years) and more male (from $38.7 \%$ to $68.3 \%$ ) population ( $\mathrm{p}$ value for gender $<0.001, \mathrm{p}$ value for change in mean age $<0.001$ ). Payer mix changed substantially at TCA and TCC, with an annual increase in uninsured patients (figure 2). TCA originally began in 1999 with $24.1 \%$ of their trauma patients as uninsured, which increased to $44.6 \%$ by 2009 . TCC saw an even more dramatic increase, with $12.9 \%$ of their trauma patients uninsured in 1999, and more than tripling to $44.6 \%$ in 2009.

Over $85 \%$ of trauma admissions in the MLK service area during the study period were African American or Latino. None of the five TCs in the study cared for a trauma patient population that was more than $15 \%$ Caucasian. TCA saw an increase of non-white patients 
Figure 1 Annual admissions, trauma centers serving MLK catchment area. MLK, Martin Luther King Jr Hospital; TC, trauma centre.

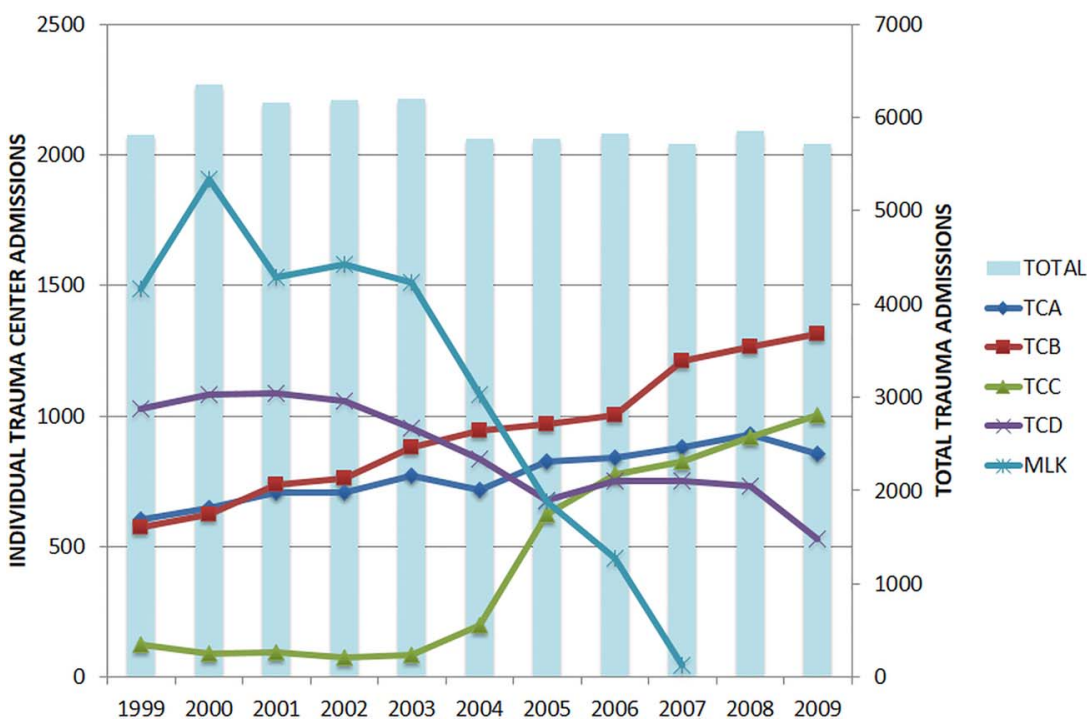

from $84.9 \%$ to $95.4 \%$ during the study period $(\mathrm{p}<0.001)$. TCB saw an increase of non-white patients from $91.9 \%$ to $97.0 \% \quad(\mathrm{p}<0.001)$. TCC saw an increase from $97.5 \%$ to $98.5 \%$ ( $\mathrm{p}=0.179)$.

\section{Injury mortality}

Unadjusted mortality

Though total trauma admissions generally remained constant during the study period, overall injury mortality for individual centres experienced wide year-to-year variations in unadjusted mortality, particularly at TCs A, B and $\mathrm{C}$, beginning with the transition to MLK closure.

\section{Adjusted mortality}

We first created a model to predict injury mortality in the MLK service area by using preclosure admissions data from all hospitals that served the area, controlling for age, race, insurance status, ISS, SRR, injury mechanism and time. Time was accounted for by using a continuous variable indicating year of the study period starting with zero for 1999. The four models had pseudo- $\mathrm{R}^{2}$ ranging from 0.085 for stabbings to 0.369 for motor vehicle collisions, and were used to predict mortality for each patient from 1999 to 2009. Observed and predicted mortality within each year of the study period were calculated by aggregating actual and predicted deaths annually. Predicted annual mortality was calculated with $99 \%$ CIs. In all models, SRRs were the variables most strongly associated with mortality. We found a small but statistically significant improvement in observed compared to predicted mortality rates from 2004 to $2009(3.0 \%$ vs $2.8 \%$ and $2.8 \%$ vs $2.4 \%)$ for the overall trauma population (figure 3 ). However, there was a statistically significant increase in GSW mortality beginning in 2004. The observed mortality increased from $5.0 \%$ to nearly $7.5 \% \quad(\mathrm{p}<0.001)$ in the years after the closure, and was nearly double the predicted mortality (figure 4). As a sensitivity analysis, we also graphed observed versus predicted mortality rates for individual centres and found the same results.

\section{DISCUSSION}

Our findings show that after the MLK closure in 2007, trauma volumes rose significantly at all adjacent hospitals except for TCD, with a marked increase in patient volumes at TCs A and C, in particular, from 1999 to 2009. The substantial redistribution of the patient load was also accompanied by an increase in the severity of injured patients at hospitals that previously cared for less severely injured patients. In addition, certain hospitals experienced an extensive shift in the payer mix of their trauma populations, with one particular trauma centre more than tripling its initial 1999 share of uninsured patients. In general, risk-adjusted mortality for trauma

Table 1 Trauma volumes by hospital and most common mechanisms, 1999-2009

\begin{tabular}{lllllc}
\hline & TCA & TCB & TCC & TCD & MLK \\
& N (\%) & N (\%) & N (\%) & N (\%) & N (\%) \\
\hline GSW & $987(22)$ & $1841(22)$ & $1522(18)$ & $1296(17)$ & $2752(33)$ \\
SW & $322(7)$ & $487(6)$ & $315(4)$ & $496(7)$ & $499(5)$ \\
MVC & $1443(32)$ & $2121(26)$ & $3272(39)$ & $2553(33)$ & $2229(27)$ \\
Falls & $1753(39)$ & $3855(46)$ & $3346(40)$ & $3280(43)$ & $2762(34)$ \\
\hline
\end{tabular}

GSW, gunshot wound; MLK, Martin Luther King Jr Hospital; MVC, motor vehicle collision and auto versus pedestrian; SW, stab wound; TC, trauma centre. 
Figure 2 MLK area trauma centers, per cent uninsured patients. MLK, Martin Luther King Jr Hospital; TC, trauma centre.

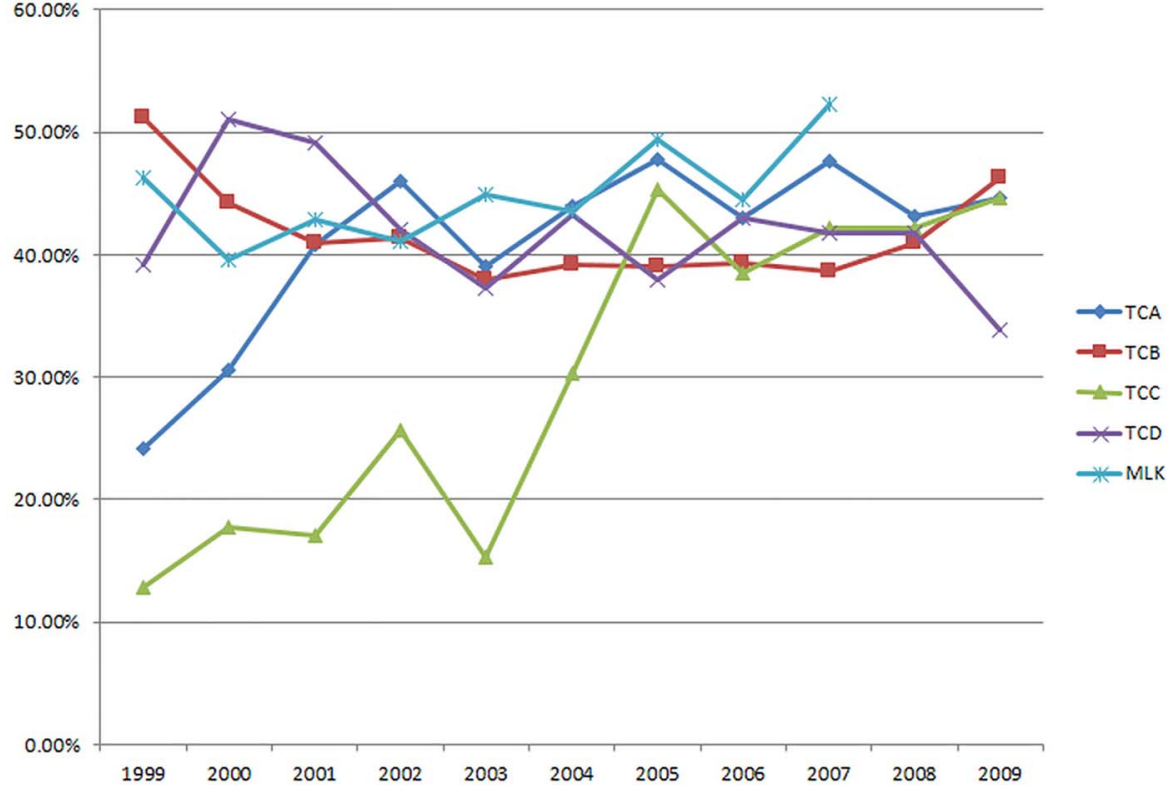

patients overall did not significantly change during the study period. However, we found a persistent and significant increase in mortality from GSWs after the closure. These findings may be explained by longer travel times and associated higher mortality for patients injured by GSWs ${ }^{26}$ random variation, or changes in weaponry, though the latter is less likely, as our results were risk-adjusted by SRR and ISS. Finally, it may be that this increased mortality after the closure indicates particular expertise of the MLK trauma centre in management of GSWs. However, given the relative lack of granularity of our risk stratification (the data set lacked ED blood pressure, transfusion data, base deficit, and other predictors of survival), we cannot make a definitive statement about this. Our conclusions cannot be necessarily extrapolated to other trauma centre closures, since this particular closure was in the context of having overlapping and well-coordinated efforts by the county to provide adequate services. Our study does show that in these types of contexts, a trauma centre closure may not necessarily lead to poorer outcomes.

Overall, violent crime rates decreased steadily during the study period, and homicide rates in LA County decreased from $9.1 / 100000$ to $6.7 / 100000$ by 2009 .

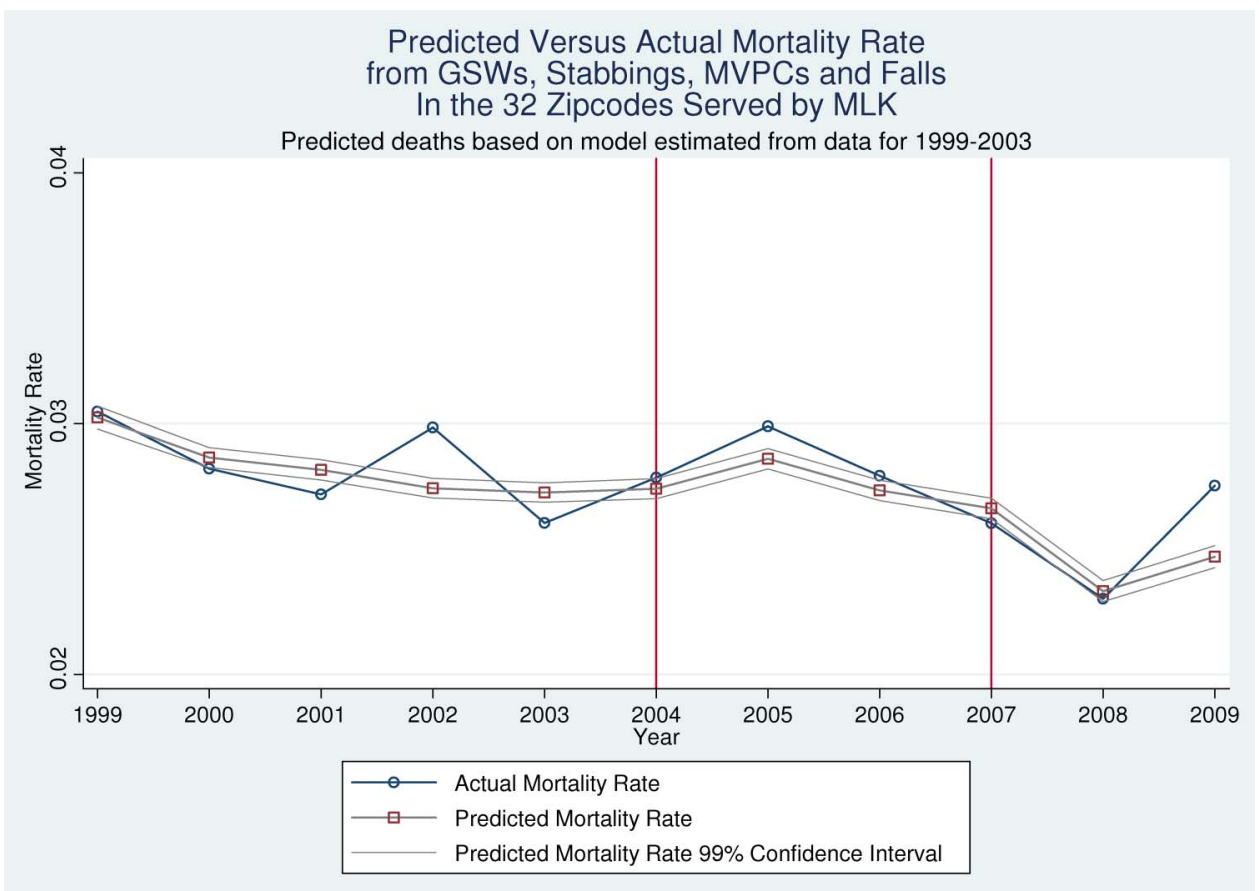

Figure 3 Overall trauma mortality rates, 1999-2009. GSWs, gunshot wounds MLK, Martin Luther King Jr Hospital. 


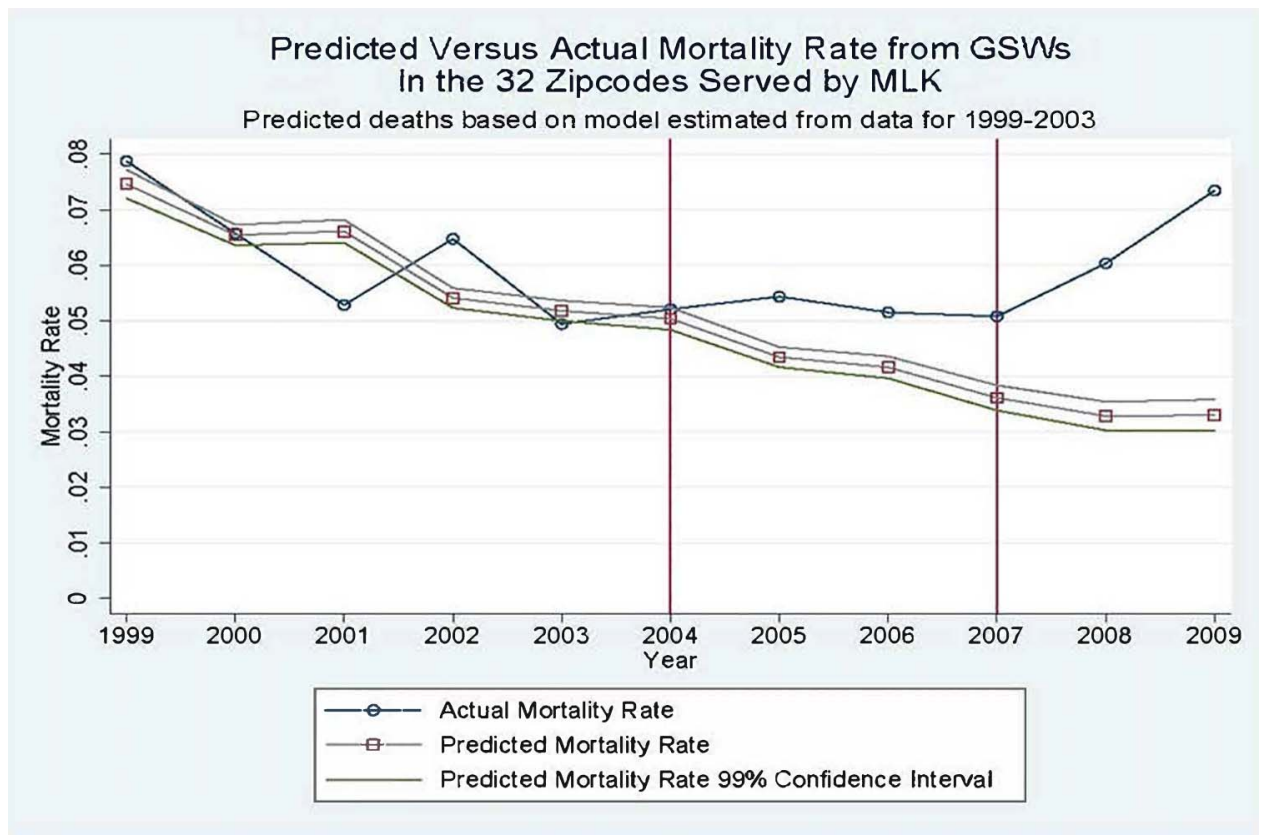

Figure 4 Trauma mortality for GSWs, 1999-2009. GSWs, gunshot wounds; MLK, Martin Luther King Jr Hospital.

Given that the homicide rate overall was decreasing, it is also possible that the increased mortality for GSW victims in south Los Angeles could be linked to MLK closure.

Our findings have several implications for policymakers and health system planners. The MLK closure was not undertaken lightly, and a transitional period of several years that included multiple efforts to improve quality outcomes was undertaken. These data demonstrate that the efforts of LA County to anticipate and forestall adverse events helped increase capacitance and decrease potentially negative impacts on trauma mortality despite dramatic increases in trauma volumes at nearby centres. An example of measures that were put in place included the creation of a new level II trauma centre in centre of the MLK catchment area (TCG) to help safeguard this vulnerable population from excess injury mortality during and after the closure. Our findings are consistent with one previous study evaluating the effects of the MLK trauma centre de-designation on Harbor-UCLA, which did not show an increase in mortality of the trauma admissions despite an increase in volume and injury severity. ${ }^{27}$ A recent paper discussing trauma closures in California overall showed that trauma patients experiencing an increased distance to the nearest trauma centre after a closure had higher inpatient mortality; ${ }^{13}$ our findings within this local context showed that this was only true for a subset of patients with GSWs. Given that penetrating injury is particularly time-sensitive, this could provide some explanation of the nuances of our findings.

This study raises an important question of whether hospital quality measures, which are aimed to serve as proxies for patient care and outcomes, are effective for evaluating trauma outcomes across hospitals. The decision to close MLK was based on its failure to meet minimal federal standards on hospital quality measures. These quality measures include many aspects of inpatient and outpatient care, including cardiovascular disease, end-stage renal disease and respiratory care. ${ }^{28}$ Though deaths and injuries related to medical errors are included in the Centers for Medicare \& Medicaid Services (CMS) appraisal, measurements of trauma care were not, and are still not included in these quality indicators. Other investigators have found that CMS quality indicators do not correlate with risk-adjusted mortality rates at trauma centres. $^{29}$ Of concern for our particular study is that GSW mortality steadily rose after redistribution of care to other hospitals, which was one of the objections voiced by opponents to the trauma centre closure. Moreover, the increase in mortality from GSWs is counter to national trends in trauma mortality and raises concerns about unanticipated effects, particularly in areas disproportionately burdened by gun violence. In the future, metrics such as those included in the TQIP, in addition to CMS quality measures, might be beneficial to help guide decisions about trauma centre closures. ${ }^{25} 30$

Finally, this study highlights the potential financial implications faced by neighbouring hospitals after a closure. It has been well-documented that 'safety-net hospitals ${ }^{\text {'31 }}$ such as MLK that provide disproportionate amounts of care to low-income and poorly insured patients are vulnerable to closure, ${ }^{12} 32$ and that trauma centres are more likely to close in areas with higher proportions of non-white individuals and a poorer payer mix. ${ }^{10} 11$ As seen in our study, the reallocation of thousands of poorly insured patients to nearby hospitals inevitably requires that they absorb the financial implications of caring for this population as well. Further research into these financial consequences, and how a health 
system can help minimise the shock on the system, is crucial.

This study has several limitations. It is a retrospective, observational study that includes only basic patient demographics and a calculated measurement of ISS. It also does not include prehospital data, such as measurements of systolic blood pressure and transport times, nor data about procedures performed, blood transfusions given or other patient-level and hospital-level data. However, it would be very difficult to undertake this type of study prospectively across multiple hospitals and a complicated EMS system in a large, urban setting. Also, we deliberately did not limit our study population to adults, in the hopes of providing the most accurate representation of the MLK patient population. However, this inclusion may have actually biased our results to the null if most injured children were being taken to other local paediatric trauma centres, which would make any volume or outcome-related changes after the closure appear smaller overall.

In addition, our findings that mortality was not impacted after the MLK closure does not take into account fatalities that did not present to a trauma centre, such as individuals pronounced dead at the scene. Furthermore, our data set does not capture deaths occurring in the ED. Because the state began collecting ED data in 2005, we are unable to include ED deaths in our study. Our findings may actually be conservative in the sense that there may be a survival bias in patients who survived the transport to hospitals that were located farther away than MLK. Last, it also may be that these findings are unique to this particular trauma system in this local context. Contextual factors likely play an important role in the impact of these closures on the surrounding communities, and our findings cannot be extrapolated to all areas of the country that have experienced closures of trauma centres.

\section{CONCLUSIONS}

Our study showed there was a significant redistribution of trauma patients to nearby hospitals after the MLK closure, with a marked increase of uninsured patients at certain hospitals. Overall, injury mortality did not change for trauma patients in south central Los Angeles, though concerns remain about the postclosure outcomes for GSW victims. Our findings shed hope on how careful planning, including using available technology such as geocoding methodology to map road traffic patterns and ambulance diversions to nearby hospitals, might anticipate potential pitfalls and inform decisionmaking with respect to resource allocation, ultimately to provide optimal patient care.

Twitter Follow Marie Crandall at @vegansurgeon

Acknowledgements The authors would like to acknowledge Sarah Sabbagh, MPH for her administrative assistance.

Contributors MC, DS, RYH, AN and XW were involved in the study concept and design. RYH was involved in the acquisition of data. MC, DS and RYH were involved in the analysis and interpretation of data. MC was involved in the drafting of manuscript. MC, DS, RYH, AN and XW were involved in the critical revision of manuscript for important intellectual content. DS was involved in the statistical analysis. MC was involved in obtained funding. MC, $\mathrm{DS}, \mathrm{RYH}, \mathrm{AN}$ and $\mathrm{XW}$ were involved in the administrative, technical or material support. MC was involved in the study supervision.

Funding This work was partly supported by a career development award from the Robert Wood Johnson Foundation Physician Faculty Clinical Scholars Programme (MC).

Competing interests None declared.

Provenance and peer review Not commissioned; externally peer reviewed.

Data sharing statement No additional data are available.

Open Access This is an Open Access article distributed in accordance with the Creative Commons Attribution Non Commercial (CC BY-NC 4.0) license, which permits others to distribute, remix, adapt, build upon this work noncommercially, and license their derivative works on different terms, provided the original work is properly cited and the use is non-commercial. See: http:// creativecommons.org/licenses/by-nc/4.0/

\section{REFERENCES}

1. Injury Center: Overview. 2010. 2011. http://www.cdc.gov/injury/ overview/

2. Rutledge R, Fakhry SM, Meyer A, et al. An analysis of the association of trauma centers with per capita hospitalizations and death rates from injury. Ann Surg 1993;218:512-21.

3. Nathens $A B$, Jurkovich GJ, Cummings $P$, et al. The effect of organized systems of trauma care on motor vehicle crash mortality JAMA 2000;283:1990-4.

4. Nathens AB, Jurkovich GJ, Rivara FP, et al. Effectiveness of state trauma systems in reducing injury-related mortality: a national evaluation. J Trauma 2000;48:25-30.

5. Buchmueller TC, Jacobson M, Wold C. How far to the hospital? the effect of hospital closures on access to care. J Health Econ 2006;25:740-61.

6. Joynt KE, Chatterjee P, Orav EJ, et al. Hospital closures had no measurable impact on local hospitalization rates or mortality rates, 2003-11. Health Aff (Millwood) 2015;34:765-72.

7. Liu C, Srebotnjak T, Hsia RY. California emergency department closures are associated with increased inpatient mortality at nearby hospitals. Health Aff (Millwood) 2014;33:1323-9.

8. Shen YC, Hsia RY. Does decreased access to emergency departments affect patient outcomes? analysis of acute myocardial infarction population 1996-2005. Health Serv Res 2012; 47:188-210.

9. Hsia RY, Kanzaria HK, Srebotnjak T, et al. Is emergency department closure resulting in increased distance to the nearest emergency department associated with increased inpatient mortality? Ann Emerg Med 2012;60:707-15.e4.

10. Hsia RY, Kellermann AL, Shen YC. Factors associated with closures of emergency departments in the United States. JAMA 2011;305:1978-85.

11. Shen YC, Hsia RY, Kuzma K. Understanding the risk factors of trauma center closures: do financial pressure and community characteristics matter? Med Care 2009;47:968-78.

12. Dailey JT, Teter H, Cowley RA. Trauma center closures: a national assessment. J Trauma 1992;33:539-46; discussion 546-7.

13. Hsia RY, Srebotnjak T, Maselli J, et al. The association of trauma center closures with increased inpatient mortality for injured patients. J Trauma Acute Care Surg 2014;76:1048-54.

14. Martin Luther King Jr. Multi-service ambulatory care center. Los Angeles County Health Services. 2011. http://www.ladhs.org/wps/ portal/King Homepage (accessed 9 Sep 2011).

15. McCone J. Report of the Governor's Commission on the Los Angeles Riots. Los Angeles, 1965.

16. Designated Trauma Centers. 2007. http://www.emsa.ca.gov/ systems/files/trauma/trma_ctr.pdf (accessed 9 Sep 2011).

17. Critical condition: examining the scope of medical services in South Los Angeles. Los Angeles: The California Endowment, 2007. http:// www.issuelab.org/resource/critical_condition_examining_the_scope_ of_medical_services_in_south_los_angeles

18. HASC Report: King-Harbor closure hospital inpatient impact analysis. Fact Sheet. Los Angeles: Hospital Association of Southern California, 2008. http://www.lahealthaction.org/library/LADHS Board_Report_and_HASCReportFactSheet032708.pdf 
19. Achieving the vision: healthcare options for Los Angeles County. Los Angeles: Los Angeles Healthcare Options Task Force, 2009. http://www.issuelab.org/resource/achieving_the_vision_healthcare_ options for los angeles county

20. Lott J. King-Harbor closure hospital inpatient impact analysis Communication to Hospital Executives, Los Angeles County Region Los Angeles: Hospital Association of Southern California, 2008. http://www.hasc.org/

21. Chernof B. Status of the implementation of the contingency services plan and Martin Luther King, Jr.--harbor hospital. Communication to the Los Angeles County Board of Supervisors. Los Angeles: Department of Health Services Los Angeles County, 2008.

22. CDC. External cause-of-injury mortality matrix based on ICD-9 cause of injury codes. Atlanta, GA: Center for Disease Control, 1997.

23. Ashkenazi I, Kessel B, Khashan T, et al. Precision of in-hospital triage in mass-casualty incidents after terror attacks. Prehosp Disaster Med 2006;21:20-3.

24. Geiger AA, deRoon-Cassini T, Brasel KJ. Considering the patient's perspective in the injury severity score. J Surg Res 2011;170:133-8.

25. Shafi $\mathrm{S}$, Nathens $A B$, Parks J, et al. Trauma quality improvement using risk-adjusted outcomes. J Trauma 2008;64:599-604; discussion 604-6.
26. Crandall M, Sharp D, Unger E, et al. Trauma deserts: distance from a trauma center, transport times, and mortality from gunshot wounds in Chicago. Am J Public Health 2013;103:1103-9.

27. Yaghoubian A, Lewis RJ, Putnam BA, et al. Impact on patient outcomes after closure of an adjacent trauma center. Am Surg 2008;74:930-4.

28. Roadmap for quality measurement in the traditional Medicare fee-for service program. Washington DC: Centers for Medicaire \& Medicaid Services, 2008. https://www.cms.gov/Medicare/Quality-InitiativesPatient-Assessment-Instruments/QualitylnitiativesGenInfo/ downloads/QualityMeasurementRoadmap_OEA1-16_508.pdf

29. Shafi S, Parks J, Ahn C, et al. Centers for Medicare and Medicaid services quality indicators do not correlate with risk-adjusted mortality at trauma centers. J Trauma 2010;68:771-7.

30. Shafi S, Nathens AB, Cryer HG, et al. The trauma quality improvement program of the American College of Surgeons Committee on Trauma. J Am Coll Surg 2009;209: 521-30.e1.

31. Baxter RJ, Mechanic RE. The status of local health care safety nets. Health Aff (Millwood) 1997;16:7-23.

32. Mansuri $\mathrm{O}$, Hoonpongsimanont $\mathrm{W}$, Vaca $\mathrm{F}$, et al. The crisis in emergency and trauma care in California and the United States. Cal J Emerg Med 2006;7:81-6. 